\title{
Effect of Active Medium Inhomogeneity on Lasing Characteristics of InAs/InP Quantum-Dash Lasers
}

\author{
Zahed M. Khan, Tien K. Ng, Udo Schwingenschlögl and Boon S. Ooi* \\ Division of Physical Sciences and Engineering \\ King Abdullah University of Science \& Technology (KAUST), Thuwal 23955-6900, Saudi Arabia. \\ *email: boon.ooi@kaust.edu.sa
}

\begin{abstract}
The authors report on the effect of quantum-dash (Qdash) inhomogeneity on the characteristics of InAs/InP Qdash laser utilizing a single state rate equation model. The inhomogeneity is assumed to follow the Gaussian approximation. From our observation, an increased in Qdash inhomogeneity results in increasing of threshold current density and redshifting of the peak lasing wavelength. The lasing linewidth of the Qdash lasers has also found to increase under large injection current, attaining a full width at half maximum (FWHM) of $\sim 17 \mathrm{~nm}$.
\end{abstract}

\section{INTRODUCTION}

Semiconductor lasers incorporating quantum confined nanostructures in the active medium has shown superior performances compared to bulk active region lasers [1]-[3]. This improvement may be attributed to the availability of discrete energy levels in the conduction and valance bands in these low dimensional structures rather than a continuous band which is the feature of a bulk semiconductor laser. These nanostructures include quantum well (Qfilm), Qdash and quantum dots (Qdot) with 1D, 2D and 3D confinement of carriers, respectively. A typical atomic for microscopy (AFM) images of Qdash and Qdot (Qbox) is shown in Fig. 1(a) and (b), respectively [1]. These structures has got attention recently because of their superior performance than Qfilm and bulk semiconductor lasers. For instance, their gain spectrum is relatively narrow and symmetric as shown in Fig 1(c) at fixed carrier injection [2]. Fig. 1(c) also includes the gain spectra of Qfilm and bulk lasers for comparison purpose, This feature finds promising applications in optical communications and networking [1]-[3]. The accomplishment of these low dimensional devices may also be ascribed to the theoretical modeling of these devices that has provided a great insight on their performance characteristics. For instance, the simulation model of quantum dot semiconductor lasers [4,5], the quantum well lasers [6] has helped in designing and fabrication of these structures for optimum performance.

Qdashes which may be treated as a dense quantum wires has also found recent attention in lasers [7]-[9] and semiconductor optical amplifiers [10]. They have been utilized in long wavelength communications with added advantages of wider gain spectrum, possible wavelength tuning [3], etc. Therefore, various theoretical models has also been developed to analyze long wavelength InAs/InP
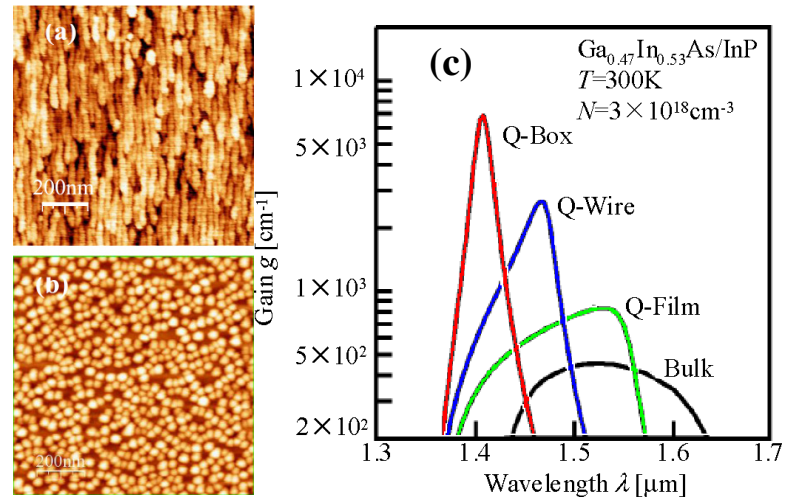

Fig. $1.1 \times 1 \mu \mathrm{m}^{2}$ AFM images of uncapped (a) Quantum dashes and (b) Quantum dots [1]. (c) Theoretical optical gain spectra of various quantum confined structures [2].

Qdash devices that are based on different techniques. For example, the density matrix formulation with rate equations [7], the energy band calculations [8,9], etc. The mail goal of this paper is to investigate theoretically the role of active medium inhomogeneity on the lasing spectra of InAs/InP Qdash laser at room temperature. The developed model utilizes the Qdash laser theory [10] that is based on solving the fundamental multi-mode carrier photon rate equations in the frame of master equation model and carrier dynamics processes [4]. The model predicted an increase in the threshold current density with increase in the active region inhomogeneity and also a red shift in the central lasing wavelength.

\section{Simulation MODEL}

The theoretical model is schematically summarized in Fig. 2 where the Qdashes are assumed to have only one confined ground state (GS, QDH) energy level. Only electron dynamics are considered in the formulation with the hypothesis that the holes being faster follows the electrons. The technique utilized here is similar to the one reported in $[4,10]$ with analogous assumptions that were made for the analysis of Qdash semiconductor optical amplifier and Qdot lasers, respectively. The reservoir of carriers, in this case, is the separate-confined heterostructure $(\mathrm{SCH})$ followed by the wetting layer $(\mathrm{WL})$. The associated time constants are $\tau_{S W}$ (relaxation from $\mathrm{SCH}$ to $\mathrm{WL}$ ), $\tau_{W D}$ (relaxation from WL to QDash), $\tau_{D W}$ (re-excitation from QDash to WL), $\tau_{W S}$ (re- 


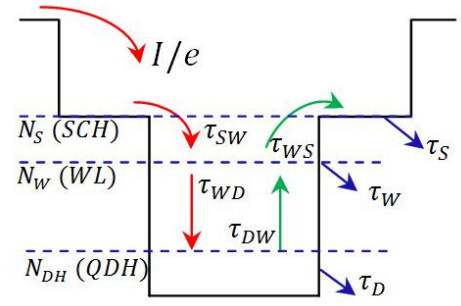

Fig. 2. Conduction band diagram of Qdash laser model including

$\mathrm{SCH}, \mathrm{WL}$ and GS energy states with their respective time constants.

excitation from WL to $\mathrm{SCH}$ ), and $\tau_{S}, \tau_{W}, \tau_{D}$ corresponding to the recombination in $\mathrm{SCH}, \mathrm{WL}$ and QDash layers, respectively. It is noteworthy to mention that, apart from interacting with each other through the WL, the QDash groups high density of states (DOS) energy tail provides another crosstalk mechanism [10]. The resulting rate equation system is as follows:

$$
\begin{aligned}
& \frac{d N_{S}}{d t}=\frac{I}{e}-\frac{N_{S}}{\tau_{S W}}-\frac{N_{S}}{\tau_{S}}+\frac{N_{W}}{\tau_{W S}} \\
& \frac{d N_{W}}{d t}=\frac{N_{S}}{\tau_{S W}}+\sum_{j} \sum_{k} \frac{N_{j, k}}{\tau_{D W}^{j, k}}-\frac{N_{W}}{\tau_{W D}}-\frac{N_{W}}{\tau_{W S}}-\frac{N_{W}}{\tau_{W}} \\
& \frac{d N_{j, k}}{d t}=\frac{N_{W} G_{j, k}}{\tau_{W D}^{j, k}}-\frac{N_{j, k}}{\tau_{D W}^{j, k}}-\frac{N_{W}}{\tau_{D}}-\frac{c \Gamma}{n_{r}} \sum_{m} g_{m}^{j, k} S_{m} \\
& \frac{d S_{m}}{d t}=\beta \sum_{j} \sum_{k} \frac{B_{j, k}^{m} N_{j, k}}{\tau_{S p}}+\frac{c \Gamma}{n_{r}} \sum_{k} \sum_{j} g_{m}^{j, k} S_{m}-\frac{S_{m}}{\tau_{p}}
\end{aligned}
$$

In the above equation system (Eqns. (1) - (4)), first two rate equation (RE) refer to the total number of carriers in the $\mathrm{SCH}\left(N_{S}\right)$ and the WL $\left(N_{W}\right)$, respectively. Eqn. (3) corresponds to the carrier dynamics in each of the intra-dash energy levels $\left(N_{j, k}\right)$ of QDash group. The subscripts $j$ and $k$ refers to the $j^{t h}$ group of QDash ensemble and its $k^{\text {th }}$ intradash energy level. These equations are then coupled with the multimode photon RE, Eqn. (4) which includes the spontaneous emission term, stimulated emission term and the photon loss. In Eqn. (4), the stimulated emission rate of photons due to $S_{m}$ is proportional to the linear gain $g_{m}^{j, k}$ calculated as the sum of the contributions of the various states at the $m t h$ mode recombination energy $E_{m}$. Therefore, the linear gain which is based on the density matrix equation, is calculated as $[3,4,8]$ :

$$
g_{m}^{j, k}=\frac{2 \pi e^{2} \hbar N_{D}}{c n_{r} \epsilon_{0} m_{0}^{2}} \frac{\left|M_{c v}\right|^{2}}{E_{c v}}\left(2 P_{j, k}-1\right) G_{j, k} B\left(E_{m}-E_{j, k}\right)
$$

The details of various terms in Eqn. (5) are reported in literature [3,8]. In brief, $g_{m}^{j, k}$ incorporates both, the homogeneous, $B\left(E_{m}-E_{j, k}\right)$ and the inhomogeneous, $G_{j, k}$, broadening. Moreover, it is worth mentioning that $N_{D}$ and $G_{j, k}$ includes the unique features of the QDash by considering the quantum wire (Qwire) like density of state (DOS) function (which is proportional to $1 / \sqrt{ } E$ ) [10]. The carrier

\begin{tabular}{|c|c|c|c|}
\hline Parameter & Description & Value & Unit \\
\hline 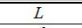 & Cavity length & $\overline{11000}$ & $\overline{\mu \mu m}$ \\
\hline$\frac{d}{d}$ & Stripe width & 40 & $\mu m$ \\
\hline$w_{W L}$ & Wetting layer thickness & 1 & $n m$ \\
\hline$w_{D H}$ & QDash width & 20 & $n m$ \\
\hline$h_{D H}$ & QDash height & 1.5 & $m m$ \\
\hline$N_{h r}$ & Number of QDash layers & 4 & \\
\hline$A_{s f f}$ & QDash effective crossection area & $1.0 \mathrm{e}-12$ & $\mathrm{~cm}^{2}$ \\
\hline$\Gamma$ & Confinement factor & 0.03 & - \\
\hline$R_{I}=R_{7}$ & Cleaved facet reflectivity & 0.3 & 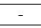 \\
\hline$\alpha_{i}$ & Internal modal loss & 10 & $\mathrm{~cm}^{1}$ \\
\hline$\beta$ & Spontaneous emission factor & $1 \mathrm{e}-4$ & - \\
\hline$N_{D}$ & QDash density of states & $5 \mathrm{e} 17$ & $\mathrm{~cm}^{3}$ \\
\hline$D_{g}$ & QDash ground state degeneracy & 1 & 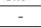 \\
\hline$D_{W}$ & WL density of states & $1.8 \mathrm{e} 19$ & $\mathrm{~cm}^{3}$ \\
\hline$E_{C V}$ & Central transition energy & 805 & $m e V$ \\
\hline$E_{W L}$ & WL ground state energy & 916 & $\mathrm{meV}$ \\
\hline$\hbar \Gamma_{\text {lom }}$ & Homogeneous broadening & 10 & $\mathrm{meV}$ \\
\hline$\tau_{S T}$ & Relaxation time from $\mathrm{SCH}$ to $\mathrm{WL}$ & 500 & ps \\
\hline$\tau_{\text {TS }}$ & $\begin{array}{l}\text { Re-excitation time from WL to } \\
\text { SCH }\end{array}$ & 1 & ns \\
\hline$\tau_{m D O}$ & $\begin{array}{l}\text { Initial capture time from WL to } \\
\text { QDash ground state }\end{array}$ & 2 & ps \\
\hline$\tau_{w}$ & Recombination lifetime of WL & 0.8 & ns \\
\hline$\tau_{D}$ & Recombination lifetime of QDash & 0.5 & ns \\
\hline$\tau_{S p}$ & Spontaneous lifetime & 2.8 & ns \\
\hline$\tau_{p}$ & Photon lifetime & 3.4 & $p s$ \\
\hline$n_{a}$ & Refractive index & 3.5 & \\
\hline
\end{tabular}
relaxation and the re-excitation rates from WL to QDash energy states are calculated according to [10] by considering
TABLE I

QDASH LASER PARAMETERS USED IN THIS SIMULATION

the initial relaxation time $\tau_{W D O}$ (capture time when the $k^{\text {th }}$ state $j^{\text {th }}$ QDash group is unoccupied).

\section{RESUlTS AND DiscUSSION}

The rate equation model Eqns. (1) - (5) has been solved in the time domain with the fourth order Runge-Kutta method up to $12 \mathrm{~ns}$ to obtain the steady state solutions. In the analysis, 701 Qdash groups with 5 intra-dash levels and 141 photon modes are considered. The parameters used in the Qdash laser simulation are summarized in Table. 1 which are obtained from the literature [10,11]. Fig. 3 shows the variation of the threshold current density and the peak threshold wavelength with the inhomogeneous broadening. It can be observed from Fig. 3(a) that large values of inhomogeneous broadening require more carriers (large threshold density value $\left.J_{t h}\right)$ in order to drive the Qdash laser to lase. This may due to more requirements of carriers by the dispersive size of the Qdashes (present in the active region) in order to satisfy the stimulated condition for lasing. Moreover, the peak threshold wavelength experiences a red shift with increase in inhomogeneity value as depicted in Fig. 3(b). This behavior may be attributed to the density of states of the Qdashes with 2D confinement. Once the lasing threshold is reached, further increase in the injected carriers resulted in broadening of the lasing spectra as shown in Fig. 4 which illustrates the lasing spectra at different current injection at a fixed inhomogeneity of $75 \mathrm{meV}$. This may due to band filling effect where the carriers start acquiring the Qdashes with high energy states once the lower energy state Qdashes are filled up. This results in short wavelength lasing in addition to long wavelength lasing. This combined effect broadens the lasing spectra and blue shifts the central lasing wavelength. This observation has been plotted in Fig. 5 at various values of current injection. It can be seen from Fig. 5(a) that the lasing wavelength shifts from $1.5608 \mathrm{~nm}$ to $1.5556 \mathrm{~nm}$ when the injection current density is increased from $1.1 J_{t h}$ to $5.0 J_{t h}$. Moreover, the lasing bandwidth which is calculated at full width at half maximum (FWHM) also increases substantially with increase in injection current. A spectral width of $\sim 17 \mathrm{~nm}$ was observed at $5.0 J_{t h}$ 

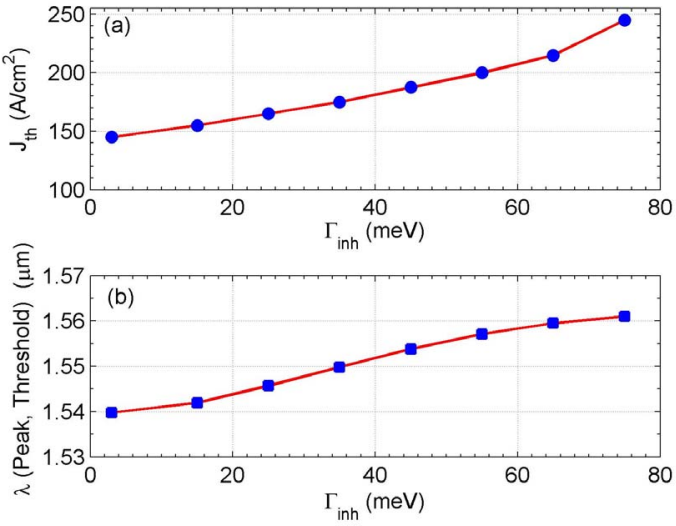

Fig. 3. Calculated (a) threshold current density and (b) threshold peak wavelength, at various active region inhomogeneity values.

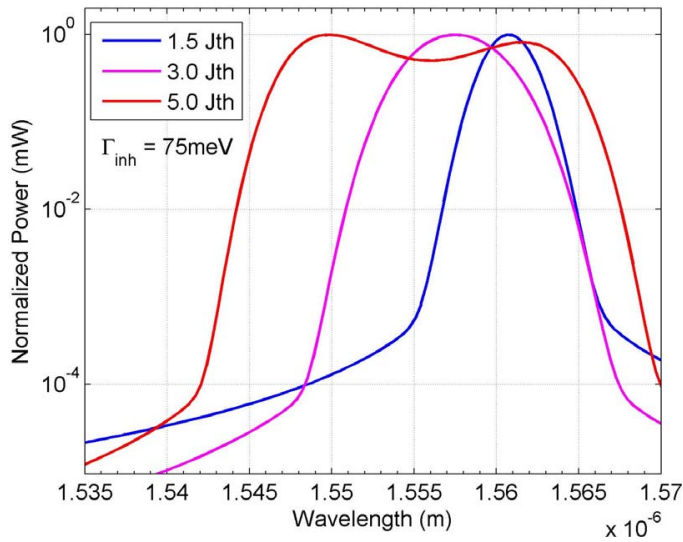

Fig. 4. Broadening of the lasing spectrum with increase in injected current density corresponding to an inhomogeneous broadening of $75 \mathrm{meV}$

corresponding to an inhomogeneous broadening of $75 \mathrm{meV}$. In the case of $25 \mathrm{meV}$ inhomogeneous broadening, a lasing bandwidth of $\sim 1.5 \mathrm{~nm}$ was observed at a current density value of $5.0 \mathrm{~J}_{t h}$.

\section{CONCLUSION}

The paper addressed a theoretical model based on rate equations for the analysis of InAs/InP Qdash lasers. The model predicted an increase in the threshold current density with an increase in the inhomogeneity of the laser active medium. In addition, the central lasing wavelength experienced a red shift on increasing the inhomogeneity value. On the other hand, increasing the current injection broadens the laser spectra along with a blue shift in the central lasing wavelength. The present model may serve as a tool to develop and optimize the performance characteristics of Qdash lasers. Presently, the model is being extended to incorporate multi-state lasing which might be helpful to provide an insight for the new class of broadband Qdash lasers reported recently by Djie et. al [12].
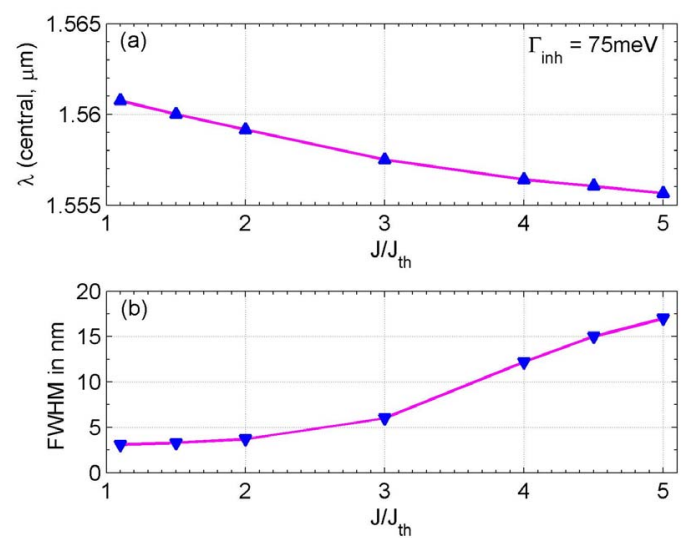

Fig. 5. Calculated (a) blue shift in the central lasing wavelength and (b) spectral width at FWHM, at various current injection values corresponding to an inhomogeneous broadening of $75 \mathrm{meV}$.

University of Michigan, Ann Arbor, under KAUST- Academic Excellence Alliance (AEA) 2010 Grant.

\section{REFERENCES}

[1] D. Zhou, R. Piron, M. Dontabactouny, E. Homeyer, O. Dehaese, T. Batte, M. Gicquel, F. Grillot, K. Tavernier, J. Even, and S. Loualiche, "Effect of stack number on the threshold current density and emission wavelength in quantum dash/dot lasers", Phys. Status Solidi, vol. C6, no. 10, pp. 2217-2221, June 2009.

[2] M. Asada, Y. Miyamoto, and Y. Suematsu, "Gain and the threshold of three-dimensional quantum-box lasers," IEEE J. Quantum Electron., vol. QE-22, no. 9, pp. 1915-1921, Sep. 1986.

[3] Z. Mi and P. Bhattacharya, "DC and dynamic characteristics of p-doped and tunnel injection 1.65 um quantum dash lasers grown on InP (001),' IEEE J. Quantum Electron. , vol. 42, no. 12, pp. 1224-1232, Nov 2006.

[4] M. Sugawara, K. Mukai, Y. Nakata and H. Ishikawa, "Effect of homogeneous broadning of optical gain on the lasing spectra in selfassembled InGaAs/GaAs quantum dot lasers," Physical Rev. B. , vol. 61, no. 11, pp. 7595-7603, March 2000.

[5] C. L. Tan, Y. Wang, H. S. Djie, and B. S. Ooi, "The spectral analysis and threshold limits of quasi-supercontinuum self-assembled quantum dot interband lasers", IEEE J. Quantum Electron., Vol. 45, pp. 1168-1176, September 2009.

[6] Qwell theoretical mod A. D. Vandermeer and D. T. Cassidy, "A Rate Equation Model of Asymmetric Multiple Quantum-Well Lasers", IEEE J. Quantum Electron. , vol. 41, no. 7, pp. 917-924, July 2005.

[7] H. Dery and G. Eisenstein, "Self-consistant rate equations of self assembly quantum wire lasers," IEEE J. Quantum Electron. , vol. 40, no. 10 , pp. 1398-1409, Oct 2004.

[8] M. Gioannini, "Numerical modeling of the emission characteristics of semiconductor quantum dash materials for laser and optical amplifiers," IEEE J. Quantum Electron., vol. 40, no. 4, pp. 364-373, April 2004.

[9] S. C. Heck, S. Osborne, S. B. Healy, E. P. O'Reilly, F. Lelarge, F. Poingt, O. L. Gouezigou and A. Accard, "Experimental and theoretical study of InAs/InGaAsP/InP quantum dash lasers," IEEE J. Quantum Electron. , vol. 45 , no. 12 , pp. $1508-1515$, Dec 2009 .

[10] D. Hadass, A. Bilenca, R. Alizon, H. Dery, V. Mikhelashvili, G Eisenstein, R. Schwertberger, A. Somers, J. P. Reithmaier, A. Forchel, M. Calligaro, S. Bansropun and M. Karkowski, "Gain and moise saturation of wide band InAs-InP quantum dash optical amplifiers: models and experiments," IEEE J Sel. Topics in Quantum Electron. , vol. 11, no. 5, pp. 1015-1026, Sep/Oct 2005.

[11] R. Schwertberger, D. Gold, J. P. Reithmaier and A. Forchel, "Long wavelength InP based quantum dash lasers," IEEE Photon. Technol. Lett., vol. 14, no. 6, pp. 735-737, June 2002.

[12] H. S. Djie, C. L. Tan, B. S. Ooi, J. C. M. Hwang, X. M. Fang, Y. Wu, J. M. Fastenau, W. K. Liu, G. T. Dang, and W. H. Chang, "Ultrabroad stimulated emission from quantum-dash laser", Appl. Phys. Lett., vol. 91, pp. 111116,2007

\section{ACKNOWLEDGMENT}

The work is supported by a joint program between KAUST and 